\title{
Wide-Field Slitless Spectroscopy with JWST's NIRISS
}

\author{
William V. Dixon ${ }^{1}$, Swara Ravindranath ${ }^{1}$ and Chris J. Willott ${ }^{2}$ \\ ${ }^{1}$ Space Telescope Science Institute, Baltimore, MD, United States \\ email: dixon@stsci.edu, swara@stsci.edu \\ ${ }^{2}$ Canadian Astronomy Data Centre, Victoria, BC, Canada \\ email: Chris.Willott@nrc-cnrc.gc.ca
}

\begin{abstract}
The Near Infrared Imager and Slitless Spectrograph (NIRISS) aboard the James Webb Space Telescope (JWST) will offer wide-field slitless spectroscopy (WFSS) with a resolving power $\mathrm{R}=150$ at wavelengths from 0.8 to 2.25 microns. In this band, NIRISS will be sensitive to Lyman $\alpha$ emission lines and continuum breaks in the spectra of galaxies with redshifts $6<$ $z<17$, allowing it to probe the first stars and ionizing sources in the early universe. NIRISS observations of the high-redshift universe will provide a wealth of information on foreground objects, creating a unique library of optical emission-line spectra from the faintest galaxies at lower redshifts. To explore its ability to identify and characterize galaxies at all redshifts, we have modeled a NIRISS observation of a massive strong-lensing galaxy cluster and analyzed the synthetic images using standard software tools. Our simulations demonstrate that WFSS with NIRISS will provide a powerful tool for the exploration of galaxies near and far.
\end{abstract}

Keywords. techniques: spectroscopic, galaxies: high-redshift, infrared: galaxies

To explore its ability to observe high-redshift galaxies - and our ability to identify them - we modeled a NIRISS observation of the massive lensing galaxy cluster MACS J0647+7015. Using published images, photometry, and redshifts from the CLASH survey (Postman et al. 2012), we constructed a series of simulated direct and dispersed images in the six filters available for WFSS with NIRISS. We added 180 simulated high-redshift $(6<z<15)$ galaxies distributed uniformly in space, redshift, and magnitude to each image. Using Source Extractor (Bertin \& Arnouts 1996), we identified 7200 galaxies in the F200W image, including 165 of our 180 high- $z$ galaxies. The 15 missing galaxies fell on bright foreground objects. We used the aXe software package (Kümmel et al. 2009) to extract and calibrate the spectra from each combination of filter and grism and an automated routine to fit a simple model to each spectrum. Of the 165 high- $z$ galaxies in our sample, 109 yield best-fit redshifts with errors $\left|z_{\text {fit }}-z\right|<0.1,5$ yield redshifts with large error bars that include the true redshift, 5 suffer from spectral overlaps that are not properly modeled by aXe, and 46 trigger an error or warning from our fitting routine. Details are presented in Dixon \& Willott (2013).

NIRISS is provided to the JWST project by the Canadian Space Agency under the leadership of René Doyon of the Université de Montréal. The prime contractor is COM DEV Canada.

\section{References}

Bertin, E. \& Arnouts, S. 1996, A\&AS, 317, 393

Dixon, W. V. \& Willott, C. J. 2013, STScI Newsletter, 31(2), 28

Kümmel, M., Walsh, J. R., Pirzkal, N., Kuntschner, H., \& Pasquali, A. J. 2009, PASP, 121, 59

Postman, M. et al., ApJS, 199, 25 